\title{
MODELS OF MEMBRANE TRANSPORT PHENOMENA AND THEIR APPLICATIONS FOR ULTRAFILTRATION DATA
}

\author{
SHIN-ICHI NAKAO AND SHOJI KIMURA \\ Institute of Industrial Science, University of Tokyo, Tokyo 106
}

\begin{abstract}
The friction model and the pore model were studied comparatively, and the latter was modified in order to remove the discrepancy between these two models. Ultrafiltration data of six solutes of various molecular weights, obtained in our previous study, were analyzed by this model, but the results were not satisfactory.

Wall correction factors were then eliminated from the modified pore model. The data were reanalyzed by this new model, the "steric hindrance-pore model", and it became clear that this model adequately interpreted the experimental data. The values for membrane pore radius obtained were in good agreement. The $A_{k} / \Delta X$ value for each solute calculated was almost constant, and agreed well with that determined from pure water permeation.

The reason why this proposed model, from which the usual wall effect is eliminated, works well is not clear at this stage.
\end{abstract}

\section{Introduction}

For the quantitative analysis of solute transport through a membrane, transport equations are necessary. At present, the equations derived phenomenologically according to nonequilibrium thermodynamics are considered most reasonable and have been generally used for analysis. In our previous study ${ }^{12)}$, we showed that these equations were also valid as transport equations of ultrafiltration.

In this analysis, characteristics of a membrane are expressed by three transport parameters, but it is impossible to clarify the mechanism of membrane permeation, because a membrane is considered to be a "black box" in nonequilibrium thermodynamics. Therefore, to illustrate the physical meaning of these parameters, several models of permeation mechanism are presented.

One of them is the friction model ${ }^{11,15)}$, in which a solute molecule is acted upon by frictional force as well as thermodynamic force. The former is defined in accordance with hydrodynamics and the latter is equal to the gradient of chemical potential, and both these forces balance in a steady flow. According to this model, characteristics of a membrane, that is, transport parameters, are expressed by three frictional coefficients, distribution coefficient of solute and volume fraction of water in the membrane.

Another is the pore model ${ }^{6,13,14,17}$, which accounts for the transport of spheres through cylindrical pores

Received September 4, 1981. Correspondence concerning this article should be addressed to $\mathrm{S}$. Nakao. impeded by steric hindrance and by frictional forces. In this model, transport parameters are expressed by radius and length of a cylindrical pore and its total cross-sectional area. Therefore, a structural interpretation of a membrane is possible if the transport parameters determined from solute rejection experiments are analyzed using this pore model, and this was attempted in our previous study ${ }^{12}$.

In the present work, the friction model and the pore model are studied comparatively, and the latter is modified in order to remove the discrepancy between these two models. Then, using this exact pore model, ultrafiltration rates and rejection of solutes are analyzed. Furthermore, wall correction factors, which express the impediment to solute flow by frictional forces, are removed from this model and its validity for analysis is investigated.

\section{Theoretical}

\subsection{Transport equations}

Kedem and Katchalsky ${ }^{10}$ phenomenologically derived the following transport equations based on nonequilibrium thermodynamics.

$$
\begin{aligned}
& J_{v}=L_{p}(\Delta P-\sigma \Delta \Pi) \\
& J_{s}=\omega \Delta \Pi+(1-\sigma) J_{v} \bar{C}_{s}
\end{aligned}
$$

Using the van't Hoff equation, $\Delta \Pi=R T \Delta C_{s}$, Eq. (2) is expressed as

$$
J_{s}=P \Delta C_{s}+(1-\sigma) J_{v} \bar{C}_{s}
$$

Instead of the average concentration $\tilde{C}_{s}$ on both sides of a membrane, Spiegler and $\mathrm{Kedem}^{16)}$ applied Eq. (3) in a differential membrane layer and integrated 
it across a membrane. The result is written as

$$
R=\sigma(1-F) /(1-\sigma F)
$$

where

$$
F=\exp \left\{-(1-\sigma) J_{v} / P\right\}
$$

In these equations, characteristics of a membrane are described by three transport parameters: pure water permeability $L_{p}$, solute permeability $\omega$ or $P$ and reflection coefficient $\sigma$. The value for $L_{p}$ is obtained from experiments of pure water permeation. The values for $\omega$ or $P$ and $\sigma$ are determined by the method of curve-fitting explained previously ${ }^{12}$.

\section{2 Friction model}

The frictional interpretation of transport parameters was first advanced by Spiegler ${ }^{15)}$ and extended by Kedem and Katchalsky ${ }^{11 .}$. In this friction model, the system considered is the flow of aqueous solution composed of solute $(s)$ and water $(w)$, through a membrane $(m)$ of thickness $\Delta X$. It is assumed that in steady flow the thermodynamic forces driving solute and water, which are the gradients of their chemical potential, are counterbalanced by mechanical frictional forces expressed as a sum of pair interactions among solute, water and membrane. According to hydrodynamics, the frictional forces are proportional to the relative velocities of the elements. Then, choosing a membrane as reference, i.e. $v_{m}=0$, the following basic equations are obtained.

$$
\begin{aligned}
& -d \mu_{w} / d x=\left(f_{w s}+f_{w m}\right) v_{w}-f_{w s} v_{s} \\
& -d \mu_{s} / d x=-f_{s w} v_{w}+\left(f_{s w}+f_{s m}\right) v_{s}
\end{aligned}
$$

Comparison of fluxes and forces with the transport parameters gives

$$
\begin{aligned}
L_{p} & =\phi_{w} \bar{V}_{w} / f_{w m} \Delta X \\
\omega & =K_{s} / \Delta X\left(f_{s w}+f_{s m}\right) \\
\sigma & =1-\omega \bar{V}_{s} / L_{p}-K_{s} f_{s w} / \phi_{w}\left(f_{s w}+f_{s m}\right)
\end{aligned}
$$

where $K_{s}=c_{s} / C_{s}$ is distribution coefficient of solute between the water in membrane and in solution.

It is hard to illustrate the physical meaning of $\sigma$ with Eq. (10). Therefore Spiegler and $\mathrm{Kedem}^{16)}$ derived the following equation.

$$
\sigma=1-\underbrace{\frac{c_{s} / C_{s}}{c_{w} / C_{w}}}_{\begin{array}{c}
\text { Exclusion } \\
\text { term }
\end{array}} \cdot \frac{1+\left(f_{w m} / f_{s w}\right)\left(\bar{V}_{s} / \bar{V}_{w}\right)}{1+\left(f_{s m} / f_{s w}\right)}
$$

Introducing Eqs. (8) and (9) into Eq. (10), we get a relationship similar to Eq. (11).

$$
\sigma=1-\underbrace{\underline{\phi}_{w}}_{\begin{array}{l}
\text { Exclusion } \\
\text { term }
\end{array}} \cdot \underbrace{\frac{f_{s w}+f_{w m}\left(\bar{V}_{s} / \bar{V}_{w}\right)}{f_{s w}+f_{s m}}}_{\begin{array}{l}
\text { Kinetic } \\
\text { term }
\end{array}}
$$

The volume fraction of water in the external solution $C_{w} \bar{V}_{w}$ is very close to unity and thus

$$
c_{w} / C_{w}=c_{w} \tilde{V}_{w} / C_{w} \tilde{V}_{w}=\phi_{w} / C_{w} \bar{V}_{w} \simeq \phi_{w}
$$

Therefore, Eq. (12) is equal to Eq. (11).

\subsection{Pore model}

Before the analysis based on nonequilibrium thermodynamics was presented, the basic pore model had been developed by several investigators ${ }^{6,13,14}$ to account for transcapillary transport of spheres. In this model, the solute flux consists of filtration and diffusion flow; both these flows are impeded by steric hindrance and by frictional forces.

Haberman and Sayre $^{9 /}$ calculated this frictional drag force of a sphere of radius $r_{s}$ moving slowly on the axis of a cylinder of radius $r_{p}$. The drag force is enhanced by the presence of the wall of a cylinder. Then,

$$
\text { Drag }=6 \pi \mu r_{s}\left\{v_{s}-v_{w} g(q)\right\} / f(q)
$$

The functions $g$ and $f$ are called wall correction factors, and are functions of $q=r_{s} / r_{p}$. These functions were expressed by Haberman and Sayre as follows.

$$
\begin{aligned}
& f(q)=\frac{1-2.105 q+2.0865 q^{3}-1.7068 q^{5}+0.72603 q_{-}^{6}}{1-0.75857 q^{5}} \\
& g(q)=\frac{1-(2 / 3) q^{2}-0.20217 q^{5}}{1-0.75857 q^{5}}
\end{aligned}
$$

Bohlin $^{4)}$ derived similar equations.

Verniory et al. ${ }^{17)}$ modified this basic pore model, using Haberman and Sayre's calculation and the friction model. Comparing Eq. (7) with Eq. (13), they obtained the following equations.

$$
\begin{aligned}
& v_{s}\left(f_{s w}+f_{s m}\right)=v_{s} f_{s w}^{0} / f(q) \\
& v_{w} f_{s w}=v_{w} f_{s w}^{0} g(q) / f(q)
\end{aligned}
$$

In these equations, $f_{s w}^{0}$ is the frictional coefficient in a free solution, given by the Nernst-Einstein equation.

$$
f_{s w}^{0}=R T / D
$$

Introducing Eqs. (16) and (17) into Eq. (7), and assuming that the chemical potential for an ideal solution may be used, the solute flow per unit cross-sectional pore area and unit time $j_{s}$, that is, $v_{s} C_{s}$, is expressed as

$$
j_{s}=-D f(q) \frac{d C_{s}}{d x}+v_{w} C_{s} g(q)-\frac{\bar{V}_{s}}{f_{s w}^{0}} C_{s} f(q) \frac{d P}{d x}
$$

Furthermore, it is assumed that water flows according to Poiseuille's law through a cylinder, i.e. $v_{w}=$ $V\left\{1-\left(r / r_{p}\right)^{2}\right\}$, and that the third term in Eq. (19) is negligible because $\bar{V}_{s} C_{s}$ very small. Thus, the solute flux is

$$
J_{s}=D \cdot f(q) \cdot S_{D} \frac{A_{k}}{\Delta X} \Delta C_{s}+J_{v} \cdot \bar{C}_{s} \cdot g(q) \cdot S_{F}
$$

where $A_{k}$ is the ratio of total cross-sectional pore area to effective membrane area, and $\Delta X$ is the pore length. $S_{D}$ and $S_{F}$ are the steric hindrance factors for diffusion and filtration flow respectively and are 
functions of $q$.

$$
\begin{aligned}
& S_{D}=(1-q)^{2} \\
S_{F}= & 2(1-q)^{2}-(1-q)^{4}=(1-q)^{2}\left(1+2 q-q^{2}\right)
\end{aligned}
$$

Comparing Eq. (20) with Eq. (3), the transport parameters $\sigma$ and $P$ are expressed as

$$
\begin{gathered}
\sigma=1-g(q) \cdot S_{F^{\prime}} \\
P=\omega R T=D \cdot f(q) \cdot S_{D}\left(A_{k} / \Delta X\right)
\end{gathered}
$$

The pure water flux is expressed by the HagenPoiseuille equation in the pore model. Thus, the pure water permeability $L_{p}$ is defined as

$$
L_{p}=\left(r_{p}^{2} / 8 \mu\right) \cdot\left(A_{k} / \Delta X\right)
$$

Relationships similar to Eqs. (23), (24) and (25) were also presented by Bean ${ }^{21}$.

\section{4 Comparison between the friction and pore models}

To understand the mechanism of transmembrane phenomena, it is very interesting to compare the friction model with the pore model.

The volume fraction of water in a membrane $\phi_{\omega}$ can be expressed as the following equation in the pore model, and is equal to $A_{k}$ :

$$
\phi_{w}=N \pi r_{p}^{2}=A_{k}
$$

where $N$ is the number of pores per unit membrane area. Thus, comparison between Eq. (8) and Eq. (25) gives

$$
\bar{V}_{w} / f_{w m}=r_{p}^{2} / 8 \mu
$$

Next, introducing Eq. (18) into Eq. (24), we get

$$
\omega=\left(A_{k} / \Delta X\right) \cdot S_{D} \cdot\left\{f(q) / f_{s w}^{0}\right\}
$$

while, in the friction model, Eq. (9) can be rewritten as

$$
\omega=\left(\phi_{w} / \Delta X\right) \cdot\left(K_{s} / \phi_{w}\right) /\left(f_{s w}+f_{s m}\right)
$$

Thus, from Eqs. (16), (26), (28) and (29), we obtain

$$
K_{s} / \phi_{w}=S_{D}=(1-q)^{2}
$$

Finally, comparison between Eq. (12) and Eq. (23) with Eq. (30) gives

$$
\frac{f_{s w}+f_{w m}\left(\bar{V}_{s} / \bar{V}_{w}\right)}{f_{s w}+f_{s m}}=\left(1+2 q-q^{2}\right) \cdot g(q)
$$

In the pore model, partial molar volume of solute can be expressed as

$$
\widetilde{V}_{s}=(4 / 3) \pi r_{s}^{3} N_{A}
$$

where $N_{A}$ is Avogadro's number. Thus, using Eqs. (16), (17), (27) and (32), the kinetic term of Eq. (12) is rewritten as

$$
\frac{f_{s w}+f_{w m}\left(\bar{V}_{s} / \bar{V}_{w}\right)}{f_{s w}+f_{s m}}=\frac{f_{s w}^{0} g(q)+(16 / 9)\left(6 \pi \mu r_{s} N_{A}\right) q^{2} f(q)}{f_{s w}^{0}}
$$

Solute radius $r_{s}$ is obtained by the Stokes-Einstein equation.

$$
r_{s}=k T / 6 \pi \mu D=R T / 6 \pi \mu D N_{A}
$$

Then, introducing Eqs. (34) and (18) into Eq. (33), we obtain

$$
\frac{f_{s w}+f_{w m}\left(\bar{V}_{s} / \bar{V}_{w}\right)}{f_{s w}+f_{s m}}=g(q)+\frac{16}{9} q^{2} f(q)
$$

Equation (35) obviously disagrees with Eq. (31). This discrepancy was pointed out by Anderson and Quinnn ${ }^{1}$, but they gave no exact solution. In this study, we remove this disagreement and express the reflection coefficient $\sigma$ in an exact form.

\subsection{Modification of the pore model}

In the above derivation of the pore model, the third term of Eq. (19) is neglected. In this section, however, we calculate it exactly with this term.

In the case of pure water permeation through a membrane, the gradient of chemical potential is expressed only by the gradient of mechanical pressure across a membrane. Thus, Eq. (6) is rewritten as

$$
-d \mu_{w} / d x=-\bar{V}_{w} d P / d x=f_{w m} v_{w}
$$

and

$$
d P / d x=-f_{w m} v_{w} / \bar{V}_{w}
$$

Introducing Eq. (36) into Eq. (19), the following equation is obtained.

$$
j_{s}=-D \cdot f(q) \cdot \frac{d C_{s}}{d x}+v_{w} C_{s}\left\{g(q)+\frac{f_{w m} \bar{V}_{s}}{f_{s w}^{0} \bar{V}_{w}} f(q)\right\}
$$

Then, assuming that water flows according to Poiseuille's law, the solute flux is expressed as

$$
\begin{aligned}
J_{s}= & D \cdot f(q) \cdot S_{D} \cdot \frac{A_{k}}{\Delta X} \cdot \Delta C_{s}+J_{v} \bar{C}_{s} \cdot S_{F} \\
& \times\left\{g(q)+\frac{f_{w m} \bar{V}_{s}}{f_{s w}^{0} \bar{V}_{w}} f(q)\right\}
\end{aligned}
$$

Comparing Eq. (38) with Eq. (2) or Eq. (3), the reflection coefficient $\sigma$ is written as

$$
\sigma=1-S_{F}\left\{g(q)+\frac{f_{w m} \bar{V}_{s}}{f_{s w}^{0} \bar{V}_{w}^{-}} f(q)\right\}
$$

and introducing Eqs. (27), (32), (34), and (18), we obtain

$$
\sigma=1-S_{F}\left\{g(q)+(16 / 9) q^{2} f(q)\right\}
$$

If one compares Eq. (39) with Eq. (35), it is clear that the discrepancy, mentioned before for the kinetic term of $\sigma$, is removed by this exact derivation. For the exclusion term of $\sigma$, we get the following relationship from Eqs. (12) and (39).

$$
\left(K_{s} / \phi_{w}\right)_{f}=S_{F}=(1-q)^{2}\left(1+2 q-q^{2}\right)
$$

Subscript $f$ denotes the flow term in Eq. (2) or (3). Equation (40) disagrees with Eq. (30), which is the exclusion term of the diffusion term, and one should pay much attention to this point.

Then, from the consideration above, we conclude that the transport parameters of $L_{p}, P$ (or $\omega$ ) and $\sigma$ are expressed by Eqs. (25), (24) and (39), respectively, in the exact pore model. The values for $\sigma$ calculated by Eqs. (23) and (39) are illustrated in Fig. 1, with 
values of $S_{F}$. The differences among these three lines are not so large.

\section{Analysis and Discussion}

The $\sigma$ and $P$ values for six solutes, polyethylene glycol (PEG $\# 4000$ ), vitamin $B_{12}$, raffinose, sucrose, glucose and glycerin, obtained in our previous study ${ }^{12)}$, are used in the following analysis.

\section{1 Analysis by the exact pore model}

In our previous study ${ }^{12)}$, we analyzed the experimental values of transport parameters with the pore model, expressed by Eqs. (23), (24) and (25). Here, we re-analyze these values with the exact pore model derived in the present study, in the following manner.

(1) The $q$ value for each solute is calculated by Eq. (39).

(2) Next, the value for $r_{p}\left(=r_{s} / q\right)$ is determined, substituting the Stokes radius of the solute.

(3) The value for $A_{k} / \Delta X$ of each solute is calculated by Eq. (24), using the $q$ value determined by step (1) and solute diffusivity in a free solution.

(4) The $A_{k} / \Delta X$ value is calculated independently by Eq. (25) with the average value of $r_{p}$ obtained in step (2), and compared with the value given in step (3).

These results are listed in Table 1 . The values for $r_{p}$ are in good agreement except for PEG $\$ 4000$, and the average value, $r_{p}=10.9 \times 10^{-8} \mathrm{~cm}$, is obtained. It follows that the values for $A_{k} / \Delta X$ are constant for a given membrane, but they increase with increase of solute radius. A similar result was obtained in our previous study ${ }^{12}$. Furthermore, the $A_{k} / \Delta X$ value given from pure water permeation disagrees with that calculated from solute permeation.

Then, the effects of two correction factors in Eq. (24), i.e. $S_{D}$ and $f(q)$, were calculated separately, and it became clear that the effect of $f(q)$ was too large for the correction. Therefore, in the next section we attempt to remove the wall correction factors from this model.

\section{2 Elimination of the wall correction factors}

The functions of $f(q)$ and $g(q)$ were originally derived by hydrodynamics in order to correct the effect of the cylinder wall. Bean ${ }^{2}$ demonstrated the validity of these functions (he employed Bohlin's relationships), using results of hydrodynamic experiments given by other investigators ${ }^{7,8)}$. The cylinders and spheres used in these experiments had large radii, i.e. $r_{p}=0.1-1.5 \mathrm{~cm}$ and $r_{s}=0.005-0.5 \mathrm{~cm}$. Thus, it is doubtful whether one can use these functions to analyze the permeation of a very small sphere through a very fine tube, and opposite results, i.e. usable and unusable, have been reported for a membrane system $^{3,5,8}$.

In the present study, we attempt to eliminate these functions from the pore model. If we can assume

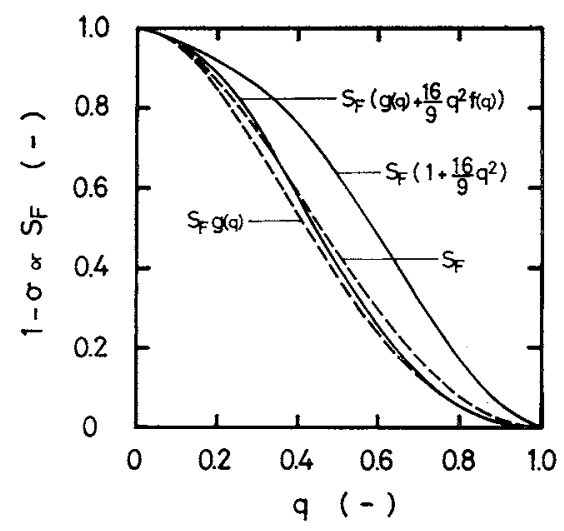

Fig. 1 Reflection coefficient as various functions of $q$

Table 1 Values of $q, r_{p}$ and $A_{k} / \Delta X$ for each solute, determined by Eqs. (39) and (24)

\begin{tabular}{llcc}
\multicolumn{1}{c}{ Solute } & $\begin{array}{c}q \\
{[-]}\end{array}$ & $\begin{array}{c}r_{p} \times 10^{8} \\
{[\mathrm{~cm}]}\end{array}$ & $\begin{array}{c}A_{k} / \Delta X \\
{\left[\mathrm{~cm}^{-1}\right]}\end{array}$ \\
\hline PEG $\$ 4000$ & 0.78 & 20.9 & 29500 \\
Vitamin $\mathrm{B}_{12}$ & 0.65 & 11.4 & 11000 \\
Raffinose & 0.54 & 10.8 & 6440 \\
Sucrose & 0.42 & 11.2 & 2470 \\
Glucose & 0.33 & 10.8 & 1460 \\
Glycerin & 0.25 & 10.3 & 2040
\end{tabular}

Average $r_{p}$ (excluding PEG $\left.\# 4000\right)=10.9 \times 10^{-8} \mathrm{~cm}$

$A_{k} / \Delta X$ calculated by Eq. $(25)=1000 \mathrm{~cm}^{-1}$

that there is very little pore-wall effect on a solute moving through the pore, we can substitute the following relationship in Eqs. (16) and (17).

$$
f_{s w}+f_{s m} \simeq f_{s w} \simeq f_{s w}^{0}
$$

Thus, Eq. (37) can be rewritten as

$$
j_{s}=-D \frac{d C_{s}}{d x}+v_{w} C_{s}\left(1+\frac{f_{w m} \bar{V}_{s}}{f_{s w}^{0} \bar{V}_{w}}\right)
$$

and the solute flux is expressed as

$$
\begin{aligned}
J_{s} & =D \cdot S_{D} \cdot\left(A_{k} / \Delta X\right) \cdot \Delta C_{s}+J_{v} \cdot \bar{C}_{s} \cdot S_{F}\left\{1+f_{w m} \bar{V}_{s} / f_{s w}^{0} \bar{V}_{w}\right\} \\
& =D \cdot S_{D} \cdot\left(A_{k} / \Delta X\right) \cdot \Delta C_{s}+J_{v} \cdot \bar{C}_{s} \cdot S_{F}\left\{1+(16 / 9) q^{2}\right\} \text { (42) }
\end{aligned}
$$

Comparison between Eq. (42) and Eq. (3) gives

$$
\begin{aligned}
& \sigma=1-S_{F}\left\{1+(16 / 9) q^{2}\right\} \\
& P=D \cdot S_{D} \cdot\left(A_{k} / \Delta X\right)
\end{aligned}
$$

Equations (43) and (44) denote "the steric hindrancepore model", from which the pore-wall effects are eliminated, and which is corrected only by the steric hindrance. There is, of course, no discrepancy between these equations and the friction model. The calculated line of $\sigma$ with Eq. (43) is illustrated in Fig. 1. There are large differences between this line and others.

The results analyzed by this new model, i.e. Eqs. (43) and (44), are listed in Table 2. The $r_{p}$ values calculated are in good agreement except for PEG \#4000. The average value, $8.4 \times 10^{-8} \mathrm{~cm}$, is smaller than that calculated by the exact pore model. It is 


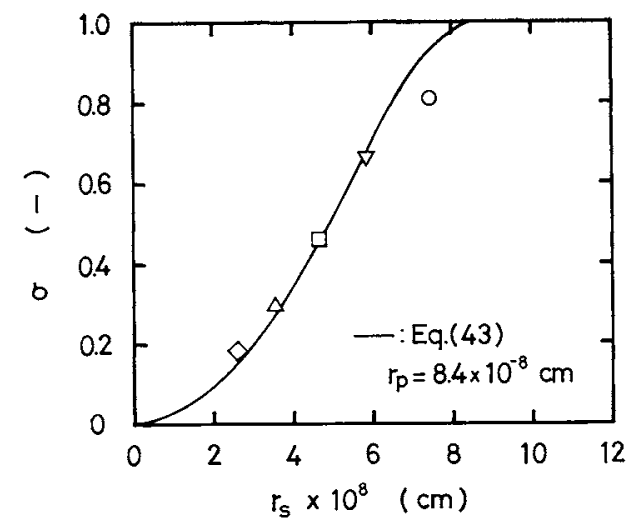

Fig. 2 Relationship between reflection coefficient and Stokes radius of solute: Keys are the same as in Fig. 3
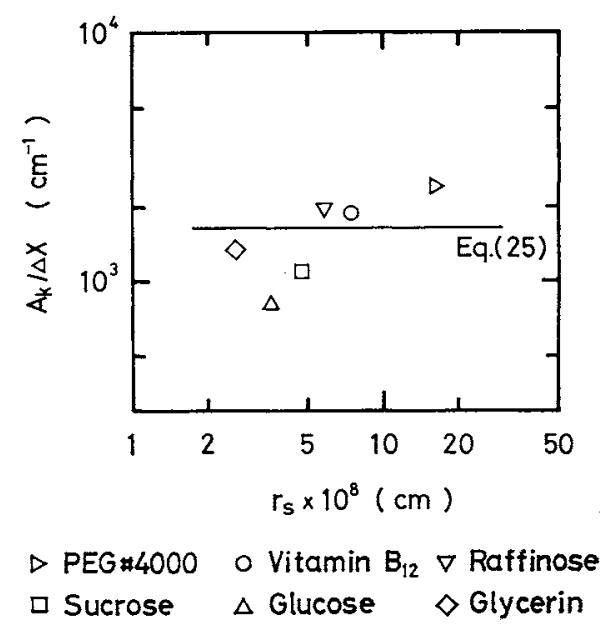

Fig. 3 Values for $A_{k} / \Delta X$ calculated by Eq. (44)

assumed that the Stokes radius is not appropriate for PEG $\$ 4000$ because it is a linear-chain polymer. The relationship between $\sigma$ and $r_{s}$ is illustrated in Fig. 2 with the theoretical line calculated by Eq. (43). The experimental data agree well with this line.

The analysis by our "steric hindrance-pore model" gives an almost constant $A_{k} / \Delta X$ value for each solute. Furthermore, as illustrated in Fig. 3, the values calculated by Eq. (44) agree very well with that determined independently by Eq. (25).

It is clear from all these results that our "steric hindrance-pore model" is the most appropriate for analysis of permeation phenomena through a membrane. The applicability of this model to other types of membranes and solutes must be determined by further experiments.

\section{Conclusion}

The friction model and the pore model, which illustrate permeation phenomena through a membrane, were studied comparatively, and it became clear that there was a discrepancy between these two models. To eliminate it, the pore model was modified
Table 2 Values of $q, r_{p}$ and $A_{k} / \Delta X$ for each solute, determined by Eqs. (43) and (44)

\begin{tabular}{lccc} 
Solute & {$\left[\begin{array}{c}q \\
-[\end{array}\right.$} & $\begin{array}{c}r_{p} \times 10^{8} \\
{[\mathrm{~cm}]}\end{array}$ & $\begin{array}{c}A_{k} / \Delta X \\
{\left[\mathrm{~cm}^{-1}\right]}\end{array}$ \\
\hline PEG \#4000 & 0.88 & 18.5 & 2410 \\
Vitamin $\mathrm{B}_{12}$ & 0.78 & 9.5 & 1880 \\
Raffinose & 0.69 & 8.5 & 1930 \\
Sucrose & 0.56 & 8.4 & 1090 \\
Glucose & 0.45 & 7.9 & 814 \\
Glycerin & 0.34 & 7.6 & 1330 \\
\hline
\end{tabular}

Average $r_{p}$ (excluding PEG $\left.\# 4000\right)=8.4 \times 10^{-8} \mathrm{~cm}$ $A_{k} / \Delta X$ calculated by Eq. $(25)=1650 \mathrm{~cm}^{-1}$

by calculating the mechanical pressure gradient term.

Ultrafiltration data of six solutes of various molecular weights were analyzed by this exact pore model, but the results obtained were not satisfactory.

Then, wall correction factors, which were assumed to express the impediment to solute flow by the effects of the pore wall, were removed from the modified model. This new model, the "steric hindrance-pore model" expressed by Eqs. (25), (43) and (44), adequately interpreted the experimental data, and gave the average value of membrane pore radius, $r_{p}=8.4 \times 10^{-8}$ $\mathrm{cm}$. The $A_{k} / \Delta X$ value for each solute calculated by this model was almost constant, and agreed well with that determined from the pure water permeation.

The reason why this proposed model works well is not clear at this stage. It is apparent that there is a definite interaction between the solute molecule and the membrane surface. The result of present work showed that this interaction cannot be attributed to the usual wall effect. In future, another form of interaction should be taken into account.

$$
\begin{aligned}
& \text { Nomenclature } \\
& A_{k} \quad=\text { ratio of total cross-sectional pore } \\
& \text { area to effective membrane area [-] } \\
& \text { C } \quad \text { concentration in solution } \quad\left[\mathrm{mol} / \mathrm{cm}^{3}\right] \\
& c \quad=\text { concentration in membrane } \\
& D \quad=\text { diffusivity } \quad\left[\mathrm{mol} / \mathrm{cm}^{3}-\mathrm{membrane}\right] \\
& f_{i j}=\text { frictional coefficient of } i \text { with } j \\
& f_{s w}^{0} \quad=\text { frictional coefficient of solute with water } \\
& \text { in free solution } \quad[\mathrm{Pa} \cdot \mathrm{cm} \cdot \mathrm{s} / \mathrm{mol}] \\
& f(q), g(q)=\text { wall correction factors for diffusion and } \\
& \text { filtration flow, respectively } \\
& J_{s} \quad=\text { solute flux through a membrane }\left[\mathrm{mol} / \mathrm{cm}^{2} \cdot \mathrm{s}\right] \\
& J_{v} \quad=\text { volume flux through a membrane }\left[\mathrm{cm}^{3} / \mathrm{cm}^{2} \cdot \mathrm{s}\right] \\
& j_{s} \quad=\text { solute flow per unit cross-sectional } \\
& \text { pore area and time } \quad\left[\mathrm{mol} / \mathrm{cm}^{2} \cdot \mathrm{s}\right] \\
& K_{s} \quad=\text { distribution coefficient of solute between water } \\
& \text { in membrane and in solution } \\
& k \quad=\text { Boltzmann's constant } \quad[\mathrm{J} / \mathrm{deg}] \\
& L_{p} \quad=\text { pure water permeability } \quad\left[\mathrm{cm}^{3} / \mathrm{cm}^{2} \cdot \mathrm{s} \cdot \mathrm{Pa}\right] \\
& N=\text { number of pores per unit membrane area }
\end{aligned}
$$




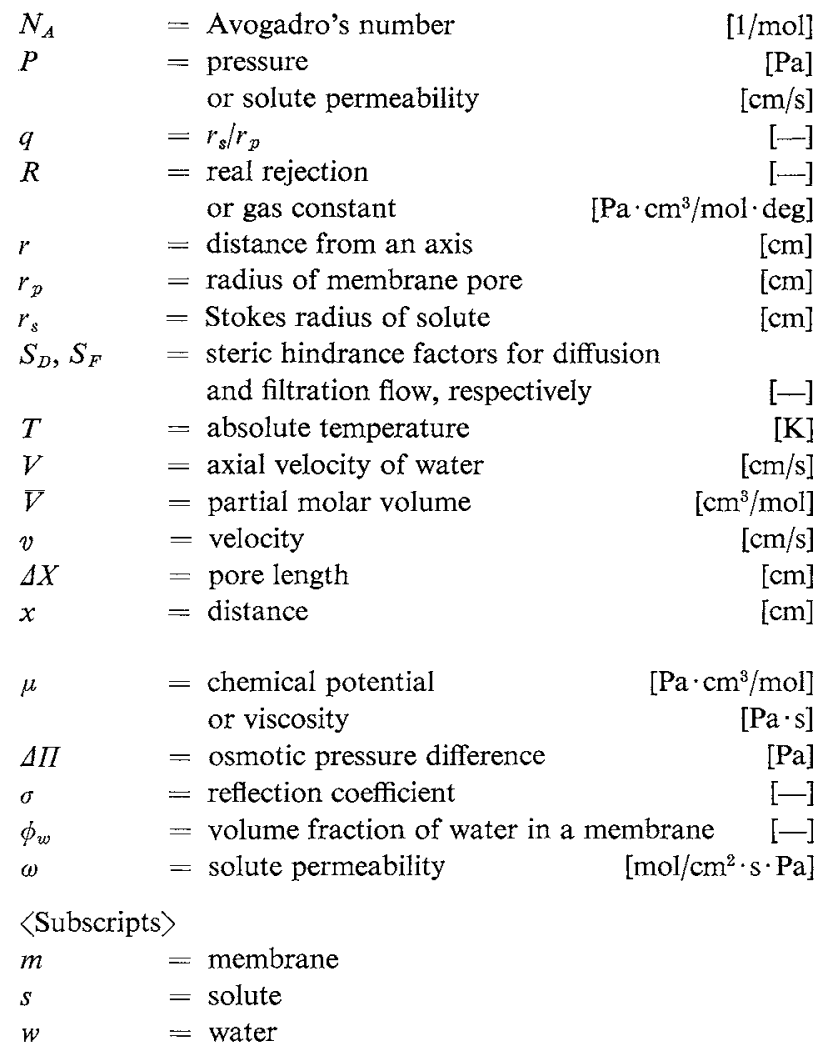

\section{Literature Cited}

1) Anderson, J. L. and J. A. Quinn: Biophys. J., 14, 130 (1974).
2) Bean, C. P.: in "Membranes-A Series of Advances, Vol. 1", G. Eisenman, ed., p. 1, Marcel Dekker, Inc., New York (1972).

3) Beck, R. E. and J. S. Schultz: Science, 170, 1302 (1970).

4) Bohlin, T.: Trans. Roy. Inst. Technol. (Stockholm), No. 155 (1959).

5) Durbin, R. P., H. Frank and A. K. Solomon: J. Gen. Physiol., 39, 535 (1956).

6) Ferry, J. D.: ibid., 20, 95 (1936).

7) Fidleris, V. and R. L. Whitmore: Brit. J. Appl. Phys., 12, 490 (1961).

8) Goldsmith, H. L. and S. G. Mason: J. Coll. Sci., 17, 448 (1962).

9) Haberman, W. L. and R. M. Sayre: David Taylor Model Basin Report No. 1143, Washington, D. C., U. S. Navy Dept. (1958).

10) Kedem, O. and A. Katchalsky: Biochim. Biophys. Acta, 27, 229 (1958).

11) idem: J. Gen. Physiol., 45, 143 (1961).

12) Nakao, S. and S. Kimura: J. Chem. Eng. Japan, 14, 32 (1981).

13) Pappenheimer, J. R., E. M. Renkin and L. M. Borrero: Am. J. Physiol., 167, 13 (1951).

14) Renkin, E. M.: J. Gen. Physiol., 38, 225 (1954).

15) Spiegler, K. S.: Trans. Faraday Soc., 54, 1408 (1958).

16) Spiegler, K. S. and O. Kedem: Desalination, 1, 311 (1966).

17) Verniory, A., R. Du Bois, P. Decoodt, J. P. Gassee and P. P. Lambert: J. Gen. Physiol., 62, 489 (1973).

18) Wendt, R. P., E. Klein, E. H. Bresler, F. F. Holland, R. M. Serino and H. Villa: J. Membrane Sci., 5, 23 (1979). 